\title{
Study on strata behavior law of weak cohesive roof in deep buried fully mechanized top coal caving face
}

\author{
Qihan Ren ${ }^{1,2, a}$ \\ ${ }^{1}$ Chongqing Research Institute CO., Ltd of China Coal Technology Engineering Group, Chongqing, China \\ ${ }^{2}$ National Key Laboratory of Gas Disaster Monitoring and Emergency Technology, Chongqing, China
}

\begin{abstract}
In view of the severe ground pressure behavior and spontaneous combustion risk of goaf in fully mechanized top coal caving face with deep buried weak cohesive roof and thick coal seam prone to spontaneous combustion, combined with theoretical analysis of mining overburden structure and field engineering practice, the structural characteristics of overburden rock and strata pressure behavior law are studied. The results show that: the deep buried thick coal seam fully mechanized top coal caving stope also has the phenomenon of surface step subsidence. Compared with the shallow coal seam, the surface subsidence is relatively slow, and the weak adhesion of the roof leads to the short periodic weighting step distance of the stope, and the average step distance is $9.9 \mathrm{~m}$. The measured peak value of advance abutment pressure is $7 \sim 10.5 \mathrm{~m}$ in front of coal wall. According to the mine pressure control and fire prevention demand of goaf, the reasonable advancing speed is $4 \sim 5 \mathrm{~m} / \mathrm{d}$.
\end{abstract}

\section{Introduction}

Zhungeer coalfield is located in Ordos Plateau, Inner Mongolia Autonomous Region. The overall trend of terrain in the coalfield is high in the West and low in the East, and the surface is covered by loess layer. The 6\# and 9\# coal seams, located in Taiyuan Formation of Upper Carboniferous system, are the main coal seams in the coal field. Most of the mining areas in the area belong to low gas mines, with large mining thickness, low gas content, easy spontaneous combustion and high $\mathrm{CO}$ content. Zhungeer coalfield has simple geological structure, gentle coal seam dip angle, stable occurrence and superior mining conditions. In the mining process of underground mining in the area, there is a phenomenon of severe ground pressure, and the working resistance of the support is generally large, which is prone to the problems of coal wall spalling, roof fall and support crushing. After trying to improve the working resistance of the support, there is still the phenomenon of frequent opening of the safety valve during the periodic weighting period ([1-4]).

Previous studies mainly focused on shallow coal seam mining. Tangjiahui coal mine is located in the southwest of Jungar coalfield, which is the deepest mine in the area. The burial depth of F61101 working face of the first mining face is more than $500 \mathrm{~m}$, which has the characteristics of severe ground pressure behavior and spontaneous combustion in goaf, and large buried depth of coal seam. In order to improve the safety and efficiency of mining, and provide scientific basis for similar working face mining, taking F61101 working face as the research object, according to the periodic weighting characteristics and basic roof weighting step distance of working face, the safe advancing speed which is conducive to mine pressure control and natural inhibition of goaf is studied, which provides guidance for safe mining of working face.

\section{Project overview}

F61101 fully mechanized top coal caving face is mainly used for mining No.6 Coal seam. The strike length of the working face is $951 \mathrm{~m}$, the dip length is $220 \mathrm{~m}$, the working face elevation is $+765 \sim 791 \mathrm{~m}$, the buried depth is $468.9 \sim 570.7 \mathrm{~m}$, and the average buried depth is $520 \mathrm{~m}$. The coal quality of No.6 Coal seam is relatively hard, containing 2 3 layers of gangue locally, and it is a nearly horizontal coal seam with a thickness of $9.64 \sim 21.7 \mathrm{~m}$ and an average coal thickness of $16.1 \mathrm{~m}$.

The main roof is mainly medium sandstone with an average thickness of $10.7 \mathrm{~m}$. It is argillaceous cemented and sub angular in shape, with poor sorting and brittle lithology. The direct roof is mainly composed of fine sandstone with an average thickness of $12.6 \mathrm{~m}$; it is locally intercalated with thin layers of sand and mudstone, with coal line streaks and brittle lithology. The direct bottom is mainly mudstone with an average thickness of $6.1 \mathrm{~m}$. The main structure of the old floor is sandy mudstone with an average thickness of $4.7 \mathrm{~m}$, and the local phase transformation is fine sandstone.

The design mining height of the working face is determined to be $4.0 \mathrm{~m}$, and the mining and caving ratio is about 1:3. The direct model of the working face is ZF $18000 / 28 / 45$, and the initial coal caving step distance is about $30 \mathrm{~m}$. The "two mining and one caving" cycle process is adopted, and the cyclic caving step distance is $1.6 \mathrm{~m}$.

${ }^{a}$ Corresponding author: 1439985797@qq.com 


\section{Numerical simulation analysis of stress evolution law of surrounding rock in stope}

The FLAC ${ }^{3 \mathrm{D}}$ model was established with the dimension of length $\times$ width $\times$ height $=600 \mathrm{~m} \times 400 \mathrm{~m} \times 218 \mathrm{~m}$. The model was solved by Mohr Coulomb plastic yield criterion. The width of the simulated working face is $220 \mathrm{~m}$ and the buried depth is $520 \mathrm{~m}$.

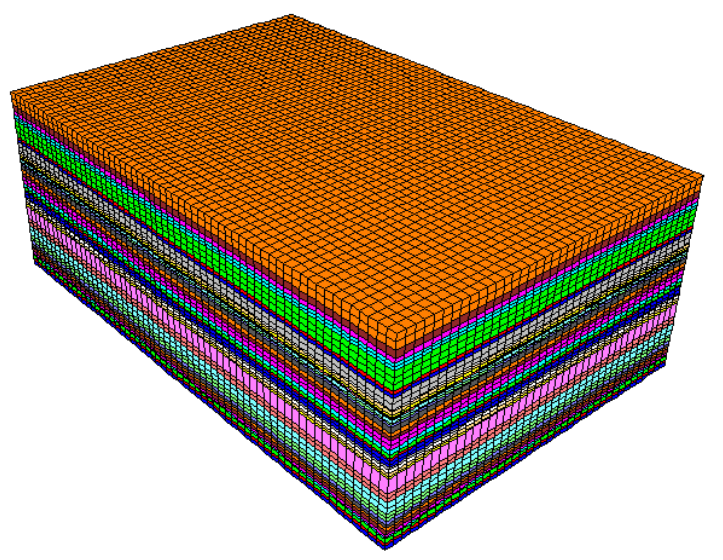

Figure 1. Model size diagram

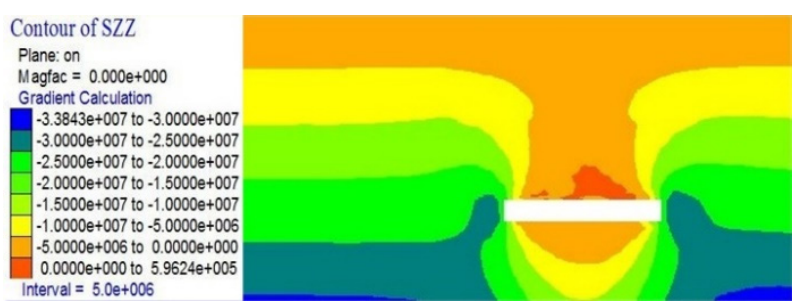

(a) Mining $120 \mathrm{~m}$



(b) Mining $160 \mathrm{~m}$

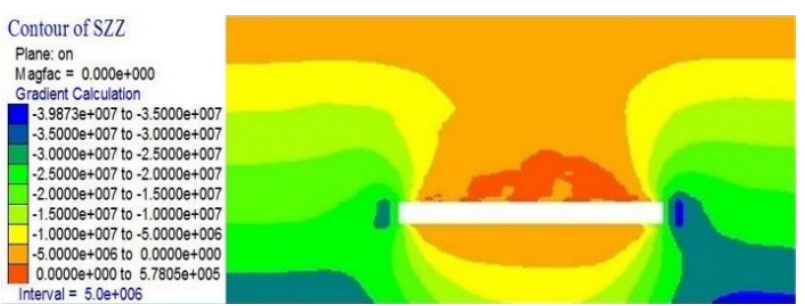

(c) Mining $200 \mathrm{~m}$

Figure 2. Stress distribution of surrounding rock along the strike direction of stope

As shown in Figure 2, a large pressure relief area is formed above the goaf, and the abutment pressure transfers to the surrounding coal and rock mass around the goaf. The stress concentration zone is formed in front of the stope and within $5 \sim 15 \mathrm{~m}$ behind the goaf. The maximum stress concentration coefficient is 1.9 .

\section{Similar simulation test of overlying strata migration in stope}

Based on the occurrence conditions of coal seam in F61101 working face, the plane stress model is selected as the test model, with geometric ratio $\mathrm{C}_{\mathrm{L}}=1: 100$, bulk density ratio $C_{\gamma}=1: 1.67$, stress ratio $C_{\sigma}=1: 167$. The size of the model is $3000 \mathrm{~mm} \times 1200 \mathrm{~mm} \times 300 \mathrm{~mm}$, and the other overlying strata are loaded according to the gravity compensation load.

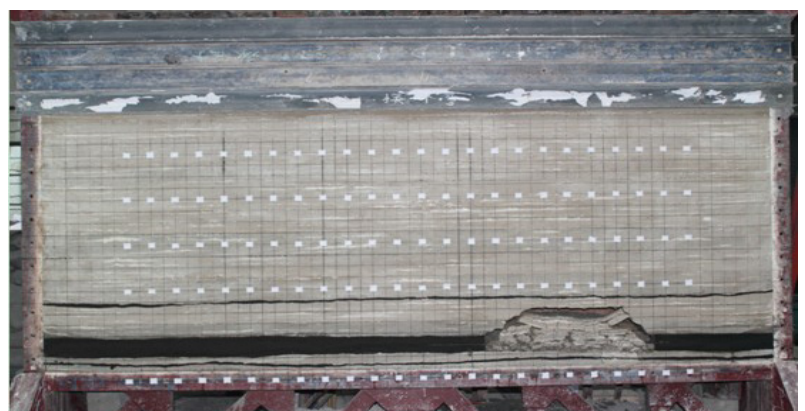

(a) Overburden caving law of working face advancing $70 \mathrm{~m}$



(b) Overburden caving law of working face advancing $130 \mathrm{~m}$

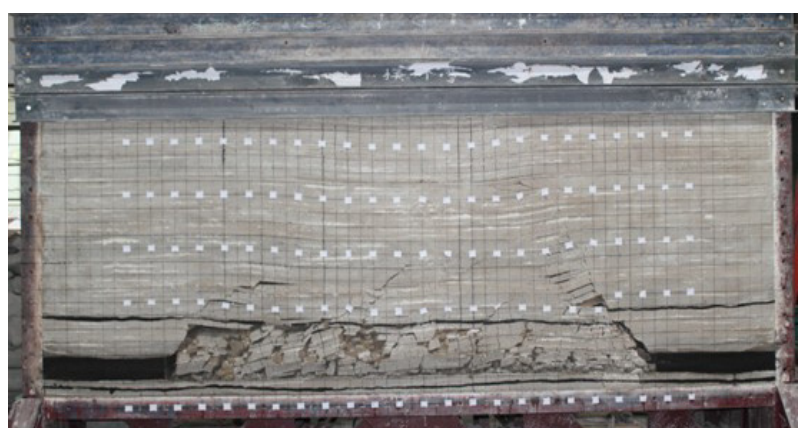

(c) Overburden caving law of working face advancing $200 \mathrm{~m}$

Figure 3. Overburden caving characteristics of stope

As shown in Figure 3, according to the characteristics of overburden caving in the advancing process of working face, after coal seam mining, the residual coal and collapsed roof can not completely fill the goaf, and the transverse and vertical fractures above the roof continue to develop. In the process of stope advancing, the roof collapses periodically, the caving height of overburden increases continuously, and the collapsed rock mass behind the goaf is continuously compacted. With the continuous expansion of the goaf, the broken line in front of the working face moves to the top of the roof. When the working face is advancing $200 \mathrm{~m}$, the front caving angle of stope is $72^{\circ}$ and the broken line is within $0 \sim 10 \mathrm{~m}$ behind the coal wall. Most of the weight of the roof will 
be directly borne by the support, and the ground pressure behavior will be more obvious.

\section{Movement characteristics of overlying strata in stope}

\subsection{Ground pressure behavior law of stope}

In the analysis of basic roof periodic weighting, the sum of the average cycle end resistance of the support and its mean square deviation is taken as the main index to judge the roof periodic weighting. The formula of data calculation is as follows ([5-7]):

$$
\sigma_{p}=\sqrt{\frac{1}{n-1} \sum_{i=1}^{n}\left(P_{t i}-\bar{P}_{t}\right)^{2}}
$$

Where: $\sigma_{P} \_$mean square error of mean value of resistance at the end of cycle; $n \longrightarrow$ measured cycle number; $P_{t i}$ The measured resistance at the end of each cycle; $\overline{P_{t}}$ _ The average value of resistance at the end of cycle.

$$
\overline{P_{t}}=\frac{1}{n} \sum_{i=1}^{n} P_{t i}
$$

Roof weighting basis:

$$
P=\bar{P}_{t}+\sigma_{p}
$$

Roof weighting started at 0:00 on December 19, and spread from the middle of the working face to both ends in turn. At the same time, during the pressure rise period from 19 to 21, the working face was accompanied by spalling of coal wall and roof watering. The initial weighting step distance was $36.9 \sim 38.3 \mathrm{~m}$, with an average of $37.6 \mathrm{~m}$ (the width of additional cut was 10.5 $\mathrm{m})$. During the weighting period of working face, the length and sequence of weighting of each part are different. No coal caving is carried out in the initial mining of $30 \mathrm{~m}$. The caving top coal has a supporting effect on the roof, and the ground pressure phenomenon is not obvious during the first weighting of the roof.

The working resistance of the hydraulic support is $8206.5 \sim 17546.6 \mathrm{KN}$ during the first weighting; The average working resistance of hydraulic support is $10609.6 \mathrm{kn}$ before and $13738 \mathrm{kn}$ when the pressure comes. The dynamic load coefficient of the first pressure is between 1.27 and 1.31.

The top coal caving results in the increase of roof rotation space. During the periodic weighting period, the working resistance of hydraulic support is higher than that of the first weighting, accompanied by coal wall spalling, and the depth and width of spalling are different. When the roof of goaf falls, large white sandstone gangue can be seen. The weighting step distance of the upper stope is $5.2 \sim 19.1 \mathrm{~m}$, the middle weighting step is $6.1 \sim 18 \mathrm{~m}$, the lower weighting step is $5.3 \sim 15.7 \mathrm{~m}$, and the average periodic weighting step distance of stope is $9.9 \mathrm{~m}$. Due to the influence of the combined support of bolt mesh and cable in the two roadways, the roof collapse is slow, which leads to the rapid weighting in the middle of the goaf, and then it develops to the direction of two lanes. During the weighting period of stope, the phenomenon of water drenching and spalling occurs.

According to the data of displacement measuring points arranged in front of the stope, the deformation of the roadway is small during the mining period. According to the stress condition of single pillar in front of the stope, the peak value of advance abutment pressure is close to the coal wall, mostly distributed in $7 \sim 10.5 \mathrm{~m}$ in front of the coal wall, and the maximum pressure peak reaches $36 \mathrm{MPa}$.

\subsection{Characteristics of land subsidence}

When the advancing length of the working face reaches $938 \mathrm{~m}$, there are cracks and bench subsidence on the surface, with the crack width of $600 \mathrm{~mm}$ and the subsidence height of $300 \mathrm{~mm}$. As shown in Figure 4, the results show that the bench sinking phenomenon is similar to that of shallow coal seam in Zhungeer coalfield, and the phenomenon appears slowly. The surface air leakage velocity is $14.31 \sim 30.28 \mathrm{~m} / \mathrm{min}$. The surface air leakage near the transportation gateway and the middle of the working face is relatively large, while the air leakage at the side of the return air gateway is relatively small.



Figure 4. Surface subsidence characteristics

\subsection{Overburden structure and instability characteristics}

Combined with the ground pressure behavior characteristics and surface subsidence characteristics of the stope, it not only has similar ground pressure behavior characteristics of adjacent shallow coal seams, such as surface step subsidence, support work resistance is large. It is also different from the roof structure characteristics formed by the mining of ordinary fully mechanized top coal caving face. The roof is mostly argillaceous and calcareous cemented filling. Under the action of various geological tectonic stresses in the later stage, the dual medium of fracture and pore is formed. The rock stratum is brittle, the uniaxial compressive strength and tensile strength are not large, and the internal cementation force is small.

The average periodic weighting step distance of stope basic roof is $5.2 \sim 19.1 \mathrm{~m}$, with an average of $9.9 \mathrm{~m}$, and 
the average thickness of basic roof is $10.7 \mathrm{~m}$. The ratio of the two is close to $0.49 \sim 1.78$, with an average of 0.93 . The cantilever length of the upper roof is short and easy to fracture, so the overlying rock can not form the "masonry beam" structure similar to the ordinary stope. The average thickness of the coal seam is $16 \mathrm{~m}$, and the caving top coal and direct roof can not fill the goaf. On the whole, the ground pressure behavior of the stope is characterized by large static pressure and small dynamic pressure.

\subsection{Reasonable advancing speed of working face}

When the advancing speed is fast, the periodic weighting step is larger, the time of top coal and roof to support is long, and the dynamic load coefficient is small. When the advancing speed is slow, the interval of periodic weighting is small, the weighting frequency is frequent and the dynamic load coefficient is large.

Table1. Comparison of ground pressure parameters under different advancing speeds of working face

\begin{tabular}{cccc}
\hline $\begin{array}{c}\text { Advancing } \\
\text { distance } / \mathrm{m}\end{array}$ & $\begin{array}{c}\text { Advancing } \\
\text { speed } / \mathrm{m} / \mathrm{d}\end{array}$ & $\begin{array}{c}\text { Weighting } \\
\text { step } / \mathrm{m}\end{array}$ & $\begin{array}{c}\text { Dynamic load } \\
\text { factor }\end{array}$ \\
\hline $0 \sim 37.6$ & 3.6 & 37.6 & 1.29 \\
$37.6 \sim 80$ & 4.1 & 9.1 & 1.47 \\
$80 \sim 125$ & 5.0 & 10.9 & 1.40 \\
$125 \sim 180$ & 4.3 & 9.5 & 1.50 \\
\hline
\end{tabular}

As shown in Table 1, the first weighting stage of stope is during $0 \sim 37.6 \mathrm{~m}$ advancing. During the first weighting, no top coal caving is carried out, and the caving coal and rock mass fill the goaf well. Combined with the reasons of bolt mesh cable support at the back of goaf, the rotary space of roof breaking during the first weighting period is small, and the ground pressure behavior is not obvious. During the $33-80 \mathrm{~m}$ advancing period, the working face experienced four times of periodic weighting, with an average periodic weighting step distance of $9.1 \mathrm{~m}$. At this stage, the advancing speed of the working face is $4.1 \mathrm{~m} / \mathrm{d}$, and the caving face starts. The top coal caving results in the increase of the rotary space of the broken roof above the goaf, and the dynamic load coefficient of the support is higher than that during the first weighting period. During the $80 \sim 125 \mathrm{~m}$ advancing period, the working face experienced four times of periodic weighting with an average interval of $10.9 \mathrm{~m}$. At this stage, the advancing speed of the working face is $5 \mathrm{~m} / \mathrm{d}$, the advancing speed of the working face is accelerated, the periodic weighting step distance is large, and the action time of top coal and broken roof on the support increases. Combined with the poor cementation of the main roof, which is easy to break and can not form a masonry beam structure, the fast advancing speed leads to the continuous increase of the support force and the continuous length of weighting.

The advancing speed not only affects the roof weighting of stope, but also requires the frame moving speed to be accelerated. The liquid supply time of the stent may be shortened, resulting in the suspension of liquid supply when the initial support force does not reach the design value. The insufficient initial support force is easy to cause roof separation and subsidence, and the coal wall is easy to spall. Considering the safety production and ground pressure control of stope, the advancing speed of working face is $4 \sim 5 \mathrm{~m} / \mathrm{d}$.

\section{Conclusion}

(1) The cantilever length of roof in fully mechanized top coal caving face of thick seam with deep weak cohesive roof is short. It is impossible to form "masonry beam" when the roof is broken periodically. The stope pressure behavior of stope generally presents the characteristics of large static pressure and small dynamic pressure.

(2) Unreasonable advancing speed and uneven coal caving are the main factors leading to abnormal release of roof pressure.

(3) According to the angle of mining pressure prevention, the safe advancing speed of working face is determined to be $4 \sim 5 \mathrm{~m} / \mathrm{d}$.

\section{References}

1. $\mathrm{Xu} \mathrm{J} \mathrm{L,} \mathrm{Zhu} \mathrm{W} \mathrm{B,} \mathrm{Ju} \mathrm{J} \mathrm{F.} \mathrm{Types} \mathrm{of} \mathrm{pressure} \mathrm{frame} \mathrm{in}$ shallow coal seam mining [J]. Acta coal Sinica, 2014, 39 (08): 1625-1634.

2. Yang P, Feng W L. Study on water and sand inrush disaster of shallow coal seam in Shenfu Dongsheng mining area $[\mathrm{J}]$. Coal science and technology, 2002 (S1): 65-69.

3. Zeng T. Study on ground pressure law of fully mechanized mining face with large mining height and shallow seam [J]. Coal engineering, 2013 (02): 46-48.

4. Yin X W, Zhu S C, Anze, et al. Determination of ground pressure law and support working resistance in shallow buried and deep fully mechanized caving face [J]. Coal science and technology, 2013, 41 (5): 50-54.

5. Hou Z J, Zhang J. Overburden fracture criterion and span calculation of shallow coal seam with thick loose bed $[\mathrm{J}]$. Journal of Liaoning University of engineering and technology, 2004 (05): 577-580.

6. Lu J, Hou Z J. Factors affecting the behavior of shallow seam pressure $[\mathrm{J}]$. Journal of mining and safety engineering, 2000, (2): 39-40, 43.

7. Liu W, Gao Z N. Similar simulation of ground pressure behavior in shallow seam mining [J]. Ground pressure and roof management, 2005, 22 (2): 17-18. 